\title{
Postmyomectomy Reproductive Outcome in Women above 35 years
}

\author{
${ }^{1}$ Krishna Kavita Ramavath, ${ }^{2}$ Srinivasa Murthy Pasumarthy \\ ${ }^{1}$ Assistant Professor, Department of Obstetrics and Gynecology, Andhra Medical College, King George Hospital \\ Visakhapatnam, Andhra Pradesh, India \\ ${ }^{2}$ Emeritus Professor, Department of Obstetrics and Gynecology, Andhra Medical College, King George Hospital \\ Visakhapatnam, Andhra Pradesh, India
}

Correspondence: Krishna Kavita Ramavath, Visiting Clinician, Department of Gynecology, Mayo Clinic, 13400 East Shea Boulevard Scottsdale, Arizona 85259, USA, Phone: 001-925-209-9568, e-mail: drkavita18@ gmail.com

\section{ABSTRACT}

The aim of this retrospective study was to assess the conception rate, reproductive outcome and complications after myomectomy in women aged above 35 years. Myomectomy was performed using abdominal and laparoscopic technique.

Myomectomy was performed in 50 women in this study. By abdominal method in 35 cases $(75 \%)$ and laparoscopic-assisted method in 15 cases $(30 \%)$. Overall conception rate is $20 \%$ which occurred after one to one and half year postmyomectomy. Multiple regression analysis showed that age was the only factor which influenced the conception rate: < 35 years, 70\% (21/30); > 35 years, 20\% (10/50; $p<0.005)$. Risks for spontaneous abortions (8\%), pre-eclampsia (10\%), preterm labor $(4 \%)$ and gestational diabetes $(10 \%)$ are increased.

All cases were delivered by cesarean section. No case of uterine rupture is seen. The retrospective study suggests that myomectomy after 35 years has only a slight increase in the reproductive performance in women presenting with infertility and has a higher risk for preeclampsia, gestational diabetes, preterm labor and NICU admission of the newborns.

Keywords: Myoma, Fibroids, Laparoscopy, Myomectomy, Infertility, Pregnancy.

\section{INTRODUCTION}

Fibroids are the commonest benign tumors of the female genital tract. ${ }^{1} \mathrm{~A}$ prevalence of $50 \%$ occurs in the reproductive age group. Not all of them are symptomatic. Only half of these women will be symptomatic in their lifetime. Fibroids are prevalent in women and increase with age in their premenopausal phase. After menopause, fibroids shrink due to lower hormonal activity, supporting the role of ovarian steroids. ${ }^{2,3}$

Fibroids are implicated as a possible cause of infertility. Other mechanisms that have been postulated for infertility and recurrent pregnancy loss are distortion of the uterine cavity, causing subacute endometritis and mechanical compression. ${ }^{4-6}$

Myomectomy is performed on symptomatic patients who desire to preserve their fertility or for infertile patients whose fibroids are picked up by the ultrasound. ${ }^{7,8}$ Removal of fibroids for fertility enhancement is done if:

- Tubo-ovarian anatomy is disturbed

- Very large sized fibroid

- Associated with increased vascularity

- Pressing any tubal ostia

- Prior to IVF/ICSI.

Date of Received: 14-01-11

Date of Acceptance: 18-04-11

Date of Publication: May 2011
Although the efficiency of myomectomy in restoring fertility has never been proved by a random clinical trial, there are several arguments indicating that myomas bear some responsibility for infertility: ${ }^{9-11}$

1. There is lower fertility rate in women presenting a myoma ${ }^{2,5}$

2. The association between myoma and infertility has been reported by two observational surveys 3,29

3. The rate of implantation for pregnancy obtained by IVF is lower in women with intramural myomas.

\section{OBJECTIVE}

Since the data concerning fertility, reproductive outcome and complications following myomectomy is inadequate in women aged above 35 years, a retrospective study of 5 years (20052009) is undertaken at King George Hospital, a government hospital affiliated to Andhra Medical College, Visakhapatnam, India, during 11 March 2008 to 10 March 2009.

\section{METHODS}

A retrospective study is done for the assessment of conception rate, reproductive outcome and complications after abdominal myomectomy and laparoscopic-assisted myomectomy (LAM) among patients admitted to the King George Hospital, Gynecology and Obstetrics Department, who are aged above 35 years (35-39 years) during 2005 to 2009. Surgical records and case sheets were checked up for relevant data and investigative work-up and operative notes. During the same 
period, relevant data for women below 35 years (25-34 years) who underwent myomectomy by similar procedures were also taken into account to match as control group.

For each patient operated in a context of infertility, a pre operative work-up was made systematically, including study of ovarian function (baseline FSH, LH, estradiol and prolactin and thyroid function tests); partner's semen analysis; transvaginal ultrasonography; examination of the uterine cavity by diagnostic hysteroscopy or hysterosalpingography. The following preoperative data were systematically collected: Characteristics of myomas existence of adnexal adhesions; tube appearance, permeability, aspect of the mucosa, existence and extent of endometriosis.

\section{Laparoscopic-Assisted Myomectomy: The Surgical Technique}

A $10 \mathrm{~mm}$ trocar with a $0^{\circ}$ laparoscope (Karl Storz, Tuttlingen, Germany) is inserted through a subumbilical curvilinear incision. Entrance into the abdominal cavity is made after pneumoperitoneum is created by veress needle.

Once the trocar is safely introduced into the abdominal cavity, the cavity was insufflated. Intra-abdominal pressure at $15 \mathrm{~mm}$ is maintained. A uterine manipulator is used to permit uterine movements through the cervix.

Three suprapubic trocars are used. One $5 \mathrm{~mm}$ trocar is inserted $3 \mathrm{~cm}$ below the umbilicus. Two other trocars are inserted in both iliac fossae, lateral to the inferior epigastric vessels. The patient is placed in $30^{\circ}$ Trendelenburg position. All the pelvic structures are visualized and abdomen is also explored in a clockwise direction. The number, size and location of the myomas are noted. The course of the ureter is traced.

Using a monopolar needle, the serosa overlying the myoma is incised and cleavage plane is identified. Myoma is fixed with a drill, and excision of the fibroid is performed. Hemostasis is secured through bipolar and the suture of the uterine wall is done through two layers. Myomas were removed and extracted from the pelvis using a morcellator, through laparoscopic port sites.

Abdomen is deflated, and incisions are closed with 4-0 Prolene or Vicryl rapid.

\section{Abdominal Myomectomy}

Laparotomy is done through a suprapubic curvilinear incision. The skin and subcutaneous tissue are opened, rectus is incised and parietal peritoneum is opened. A 3 to $6 \mathrm{~cm}$ linear inciscion is performed on the most prominent part of the myoma. Enucleation was done after identifying pseudocapsule. Careful dissection would help to enucleate and bleeders are coagulated and desiccated. Hemostasis is secured trough bipolar. The uterine wall is closed in one or two layers with interrupted sutures. The abdominal incision is closed with mattress sutures.

All the patients had antibiotic prophylaxis (cefoxitin 1gm IV) preoperatively. All the laparoscopic procedures and most of the abdominal myomectomies were performed under general anesthesia.
The variables used for analysis were the following: Age, duration of infertility, primary or secondary infertility; existence of uterine cavity deformation; total number of myomas; type (pedunculated, sessile or intramural), size (greatest diameter) and location of the largest myoma existence of a male factor and existence of an ovulatory factor (anovulatory cycles low or normal FSH, or hyperprolactinemia, or polycystic ovary); existence of tubal pathology (hydrosalpinx, phimosis, proximal anomalies); tubo-ovarian adhesions (whatever the degree of severity); existence of endometriosis according to the revised American Fertility Society classification; history of surgery for infertility associated with the myomectomy and, if so, what type. A written questionnaire was given to those traced, in the regional language and asked to fill-up information about their postoperative period.

\section{RESULTS}

The results are expressed as means \pm SD. Parameters before and after myomectomy were compared by student's t-test. Results were analyzed by $2 \times 2$ contingency table analysis and stepwise multiple regression analysis with the help of the departmental biostatistician.

Myomectomy was successfully performed in all 50 women in the study. Mean duration of follow-up is 2 years (20-33 months). Abdominal myomectomy in 35 cases (70\%) and laparoscopic-assisted myomectomy (LAM) in 15 cases (30\%) was performed in 50 patients aged over 35 years as a fertility enhancing procedure (Fig. 1).

Overall, 10 of 50 (20\%) subjects conceived after myomectomy. The possible impact of age (35 or 36 years), infertility, location and number of fibroids on the outcome was further analyzed by contingency table analysis (Table 1). Women aged $<35$ years had a significantly $(\mathrm{p}<0.005)$ higher chance of conception (70\%) than women aged $>35$ years (20\%). A history of infertility also influenced the chance of conception after myomectomy. Those without a history were significantly more likely to conceive than those with a history of infertility. The location, number and size of fibroids did not appear to have any significant impact on the conception rate.

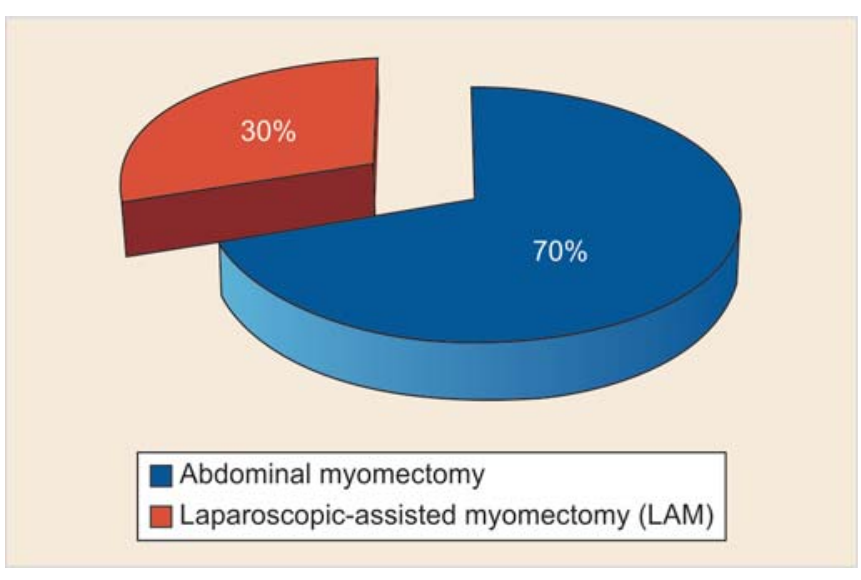

Fig. 1: Type of myomectomy 
Table 1: Impact of age and nature of fibroids on the reproductive outcome

\begin{tabular}{llcrrr}
\hline & & Live birth & Miscarriage & Total pregnant & $p$-value \\
\hline Age (years) & $<35$ years (25-34) & 15 & 6 & 21 & $<0.005$ \\
& $>35$ years & 8 & 4 & 12 & $<0.005$ \\
Overall size of & Small & 5 & 2 & 7 & NS \\
fibroid/uterus & Big & 16 & 5 & 21 & NS \\
Location of fibroid & Intramural (12 cases) & 2 & 2 & 6 & NS \\
(above 35 years) & Subserosal (23 cases) & 5 & 1 & 2 & NS \\
& Subserosal and intramural & 1 & 1 & & \\
& (15 cases) & & & & \\
\end{tabular}

NS: Not significant

\section{Patient Characteristics}

Patient characteristics are reported in Table 2.

Among these, 42 patients (84\%) were traced after collecting the data from the records and written answers were analyzed and a detailed interview was taken. Though eight of the patients were included in the study, five of them no longer came for the follow-up as they could not be traced. One of them had bilateral tubal obstruction, second had azoospermia in the partner, and the third case had anovulation with high FSH levels.

These patients demonstrated a conception rate of $20 \%$ after myomectomy and majority of these conceptions occurred within 1 to 1 and 1/2 year of the surgery. Median duration of followup for patients who did not conceive was 2 years. The outcome of pregnancies is reported in Table 3.

The average number of removed myomas was $2.2 \pm 1.7$. The largest myoma was measuring $6.5 \pm 2.2 \mathrm{~cm}$.

Twenty-three (46\%) out of the 50 cases presented with subserous myomas. Fifteen (30\%) presented with subserous intramural and 12 (24\%) of them with intramural myomas. None of the fibroids had involved or distorted the uterine cavity. A fibroid was considered subserosal, if $>50 \%$ of the fibroid protruded out of the serosal surface of the uterus.

Around 35 patients (70\%) tried for a pregnancy; 28 of them (56\%) needed a new intervention like IUI/IVF postoperatively.

Out of them only 10 patients became pregnant after myomectomy.

Table 2: Features of patients in the study

\begin{tabular}{lrr}
\hline & $n$ & $\%$ \\
\hline Myomectomy done & 50 & 100 \\
Lost to follow-up & 5 & 10 \\
Tried to get pregnant & 35 & 70 \\
Pregnant patients & 10 & 20 \\
No. of pregnancies & 10 & 20 \\
\hline
\end{tabular}

Table 3: Outcome of pregnancies

\begin{tabular}{lrr}
\hline & $n$ & $\%$ \\
\hline No. of pregnancies after myomectomies & 10 & 20 \\
Pregnancies after abdominal myomectomies & 5 & 10 \\
Pregnancies after laparoscopic myomectomies & 5 & 10 \\
Spontaneous abortion & 4 & 8 \\
Preterm labor & 2 & 4 \\
Deliveries & 6 & 12 \\
\hline
\end{tabular}

Out of 35 cases of abdominal myomectomy, five cases (14\%) obtained pregnancy and with laparoscopic myomectomy five more cases (33\%) obtained pregnancy.

Among the 50 study group, 15 women had primary infertility whereas 10 women had secondary infertility; 10 (20\%) conceived following myomectomy. All women who conceived in this group were $<40$ years of age (mean age 37 years). Of the subjects who did not conceive, 12 were of 39 years of age. Consequently, the conception rate for women $>35$ to $<40$ years was $20 \%$ (10/50). Figure 2 shows the conception rates in both the types of myomectomy.

Out of the 35 who tried to obtain pregnancy, eight of them had spontaneous conception. Of the 28 (56\%), who tried to achieve pregnancy with alternate methods, like IVF/ICSI, two concieved after undergoing IVF at private hospitals.

Out of the 10 (20\%) patients who obtained pregnancy, four (8\%) had spontaneous abortions.

Two patients (4\%) presented with the risk of abortion at 12 weeks and they subsequently presented as preterm labor at 32 weeks, and continued with tocolysis. They underwent cesarean section at completed 37 weeks, and six (12\%) had delivery by cesarean section. All the cesareans were elective mainly because of the age of the patient, length of infertility, presence of a uterine scar and pregnancy complications.

Five $(10 \%)$ patients had developed pre-eclampsia and gestational diabetes. Figure 3 shows the complications during pregnancy.

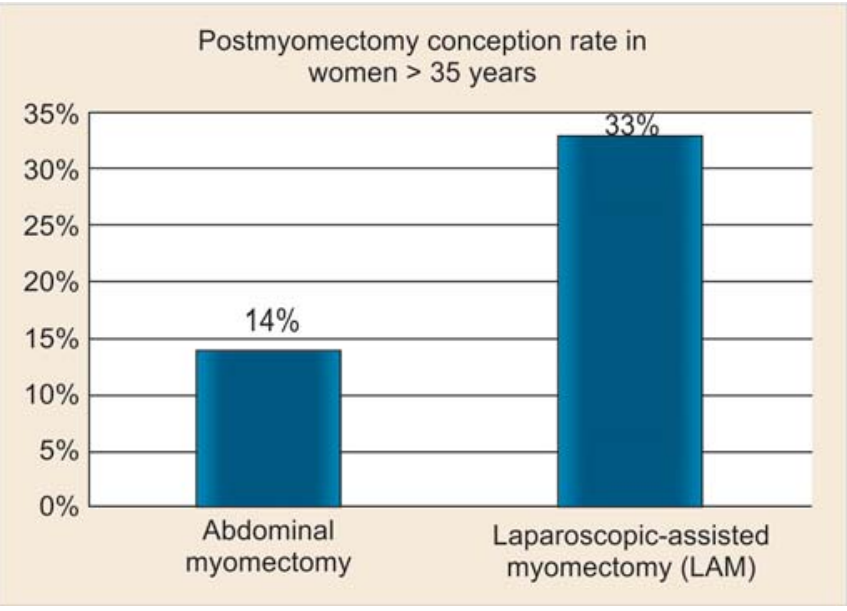

Fig. 2: Conception rates with abdominal myomectomy and laparoscopic-assisted myomectomy 
All the babies delivered by cesarean section were admitted in NICU for a couple of days and were returned to the mothers after reaching optimum weights.

The results are:

- Infertility seen in $80 \%$

- Lost to follow-up $12 \%$

- Pregnancy rate $20 \%$

- Abortion rate 8\%

- Scar rupture $0 \%$

- Cesarean section-all those who delivered

- Normal delivery $0 \%$.

No uterine rupture or dehiscence of the uterine walls is noted. Figure 4 shows postmyomectomy results of the study.

\section{DISCUSSION}

Fertility after myomectomy in women aged above 35 years is lower. Multiple factors can be taken into account, like tubo-ovarian adhesions and tubal factors. ${ }^{12-14}$ Drawbacks of the study are:

- Sample size is small

- As it is a retrospective study, it could not gather many relevant preoperative and postoperative history

- Preoperative uterine cavity assessment was not done in few cases as surgeons were not the same always

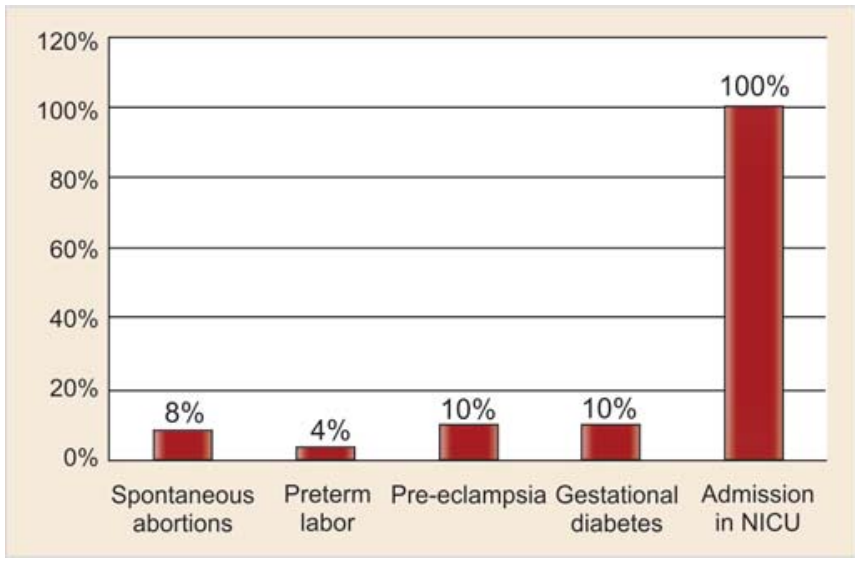

Fig. 3: Complications during pregnancy

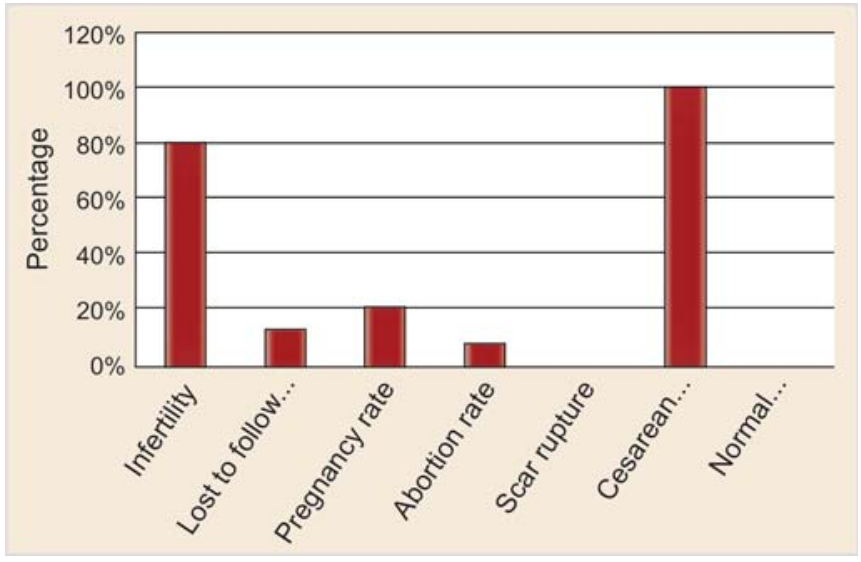

Fig. 4: Postmyomectomy results in women $>35$ years
- Data on postoperative fertility is collected through oral questionnaire.

Intramural myomas themselves are known to hamper fertility. ${ }^{15-17}$ However, in this study, the fertility after myomectomy for intramural myomas was lower than that of other types of myomas. This result can be explained by the fact that these intramural myomas were associated with an increased risk of postoperative adhesions.

The distortion and enlargement of the endometrial cavity by submucous and intramural leiomyomas with an intracavitary component affect implantation. ${ }^{18}$ Implantation failure may also be explained by focal endometrial vascular disturbances, endometrial inflammation and secretion of vasoactive substances. ${ }^{19}$ Leiomyomas may also cause dysfunctional uterine contractility and interfere with sperm and ovum transport. Likewise, intramural leiomyomas may also obstruct tubal ostia.

While many women with fibroids conceive, some may not. Some studies have demonstrated laparoscopic myomectomy had benefits higher than abdominal myomectomy. ${ }^{20-22}$ Myomectomy operations frequently result in pelvic adhesions, which may impair fertility. ${ }^{23,24}$

The main concerns after LAM are high rate of abortion, premature delivery and high rates of cesarean section. Despite these adverse events, more than half of the patients become pregnant after the surgery. ${ }^{1}$

In this study, we wanted to evaluate the conception rates at the age of 35 and above, after myomectomy. Women aged $<35$ years had a significantly $(\mathrm{p}<0.005)$ higher chance of conception after myomectomy than women $>35$ years. The stepwise multiple regression analysis showed that the chance of conception following myomectomy was primarily influenced by age. A history of infertility did not significantly influence the chance of conception after the age fact had been taken into consideration. We have demonstrated that both the procedures did not show any major difference in achieving conception rates. Review literature demonstrates ${ }^{25}$ that most of the patients conceive between 12 and 36 months after surgery and our study also showed a rate very similar. In the study, abortion rate $8 \%$ is lower than that reported by Dubuission et al. ${ }^{7}$ The majority of abortions occurred during first trimester just as other investigators reported. ${ }^{26}$ The number, location and size did not significantly affect the miscarriage rate following myomectomy.

Uterine fibroids cause many complications in the third trimester, like premature rupture of membranes, intrauterine growth retardation, postpartum hemorrhage. ${ }^{27,28}$ Many researchers demonstrated that high percentage of cesarean sections after myomectomy. ${ }^{28}$ We too preferred a cesarean section, mainly due to the fact of advanced age and number of infertile years. As a matter of fact, we wanted to minimize maternal and fetal risks also.

Complications like gestational hypertension, preterm labor, gestational diabetes pre-eclampsia were more common in the aged antenatal, ${ }^{29}$ and our study showed pre-eclampsia and gestational diabetes in $10 \%$ of the study population who are aged $>38$ years. 
No cases of uterine rupture are seen in our study. Age appeared to have a clinically significant impact on pregnancy. ${ }^{30}$ The nature of fibroid did not affect as successful removal restored the normal uterine anatomy.

In our study, the reproductive outcome of women aged above 35, following myomectomy is low. Age and multiple coexisting factors lower the pregnancy rate.

\section{CONCLUSION}

In patients with infertility due to fibroids, myomectomy is the preferred mode of treatment. Pregnancy rates postmyomectomy are variable. After the age of 35 years, several factors that were proposed, like nulliparity and infertility, seem to be poor factors for conceiving but significantly did not influence the conception. Myomectomy is beneficial for patients who are infertile due to uterine cavity distortion. Women above 35 years are more susceptible to pre-eclampsia, gestational diabetes and preterm labor. Babies born to these women have increased NICU admission.

\section{REFERENCES}

1. Sudhik R, Husch K, Steller J, Erhard D. Fertility and pregnancy outcome after myomectomy in sterility patients. Eur J Obstet Gynecol 1996:65:209-14.

2. Parazzini F, La Vecchia C, Negri E, et al. Epidemiologic characteristics of women with uterine fibroids: A case control study. Obstet Gynecol 1988;72:853-57.

3. Parazzini F, Negri E, La Vecchia C, et al. Reproductive risk factors and risk of uterine fibroids. Epidemiology 1996;7: 440-42.

4. Paulson R. Letter to the editor. Fertil Steril, 59, 1332. Rosenfeld, DL (1986). Abdominal myomectomy for otherwise unexplained infertility. Fertil Steril 1993;46:328-30.

5. Ross RK, Pike MC, Vessey MP, et al. Risk factors for uterine fibroids: Reduced risk associated with oral contraceptives. Brit Med J 1986;293:359-62.

6. Sehgal N, Haskins AL. The mechanism of uterine bleeding in the presence of fibromyomas. Am J Surg 1960;26.

7. Dubbuisson JB, Fauconnier A. Reproductive outcome after laparoscopic myomectomy in infertile women. J Reprod Med 2000;45:23-31.

8. Dubuisson1 JB, Fauconnier A, Ancel PY, Chapron C Paris, France Ozeren M, Ulusoy M, Uyanik E. First-trimester spontaneous uterine rupture after traditional myomectomy: Case report. Isr J Med Sci 1997;33:752-53.

9. Smith DC, Uhlir JK. Myomectomy as a reproductive procedure. American Journal of Obstetrics and Gynecology 1990;162: 1476-79.

10. Starks GC. $\mathrm{CO}_{2}$ laser myomectomy in an infertile population. J Reprod Med 1988;33:184-86.
11. Stovall D, Parrish S, Van Voorhis B, et al. Uterine leiomyomata reduce the efficacity of assisted reproduction cycles: Results of a matched follow-up study. Hum Reprod 1998;13:192-97.

12. Tan SL, Royston P, Campbell S, et al. Cumulative conception and live birth rates after in vitro fertilization. Lancet 1992;339: 1390-94.

13. Tulandi T, Murray C, Guralnick M. Adhesion formation and reproductive outcome after myomectomy and second look laparoscopy. Obstet Gynecol 1993;82:213-15.

14. Vercellini P, Maddalena S, De Giorgi O, et al. Determinants of reproductive outcome after abdominal myomectomy for infertility. Fertil Steril 1999;72:109-14.

15. Verkauf BS. Myomectomy for fertility enhancement and preservation. Fertil Steril 1992;58:115.

16. Rice JP, Kay HH, Mahony BS. The clinical significance of uterine leiomyomas in pregnancy. Am J Obstet Gynecol 1989;160:1212-16. Medline Web of Science.

17. Talia Eldar-Geva, Simon Meagher. Effect of intramural, subserosal and submucosal uterine fibroids on the outcome of assisted reproductive technology and treatment. Fertil Steril 1998;70:5-9.

18. Rosenfeld DL. Abdominal myomectomy for otherwise unexplained infertility. Fertil Steril 1986;46:328-33.

19. Patient C, Prentice A, Sutton DJG, et al. Myolysis of a cervical fibroid with an Nd:YAG laser. Br J Obstet Gynaecol 1996;103:584-85. Medline Web of Science.

20. Ramzy AM, Sattar M, Amin Y, et al. Uterine myomata and outcome of assisted reproduction. Hum Reprod 1998;13: 198-202.

21. Reyniak JC, Coernthal L. Microsurgical laser technique for abdominal myomectomy. Microsurgery 1987;8:92-98.

22. Ugur M, Turan C, Mungan T, et al. Laparoscopy for adhesion prevention following myomectomy. Int J Gynecol Obstet 1996;53:145-49.

23. Vercellini P, Maddalena S, De Giorgi O, et al. Abdominal myomectomy for infertility: A comprehensive review. Hum Reprod 1998;13:873-79.

24. Buttram VC Jr, Reiter RC. Uterine leiomyomata: Etiology, symptomatology, and management. Fertil Steril 1981;36: 433-45.

25. Garcia CR, Tureck RW. Submucosal leiomyomas and infertility. Fertil Steril 1984;42:16-19.

26. Dubuisson JB, Nezhat, et al. Laparoscopic myomectomy: A current view. Hum Reprod 6:588-94.

27. TC Li, Mortimer R, Cooke ID. Myomectomy: A retrospective study to examine reproductive performance before and after surgery. Hum Reprod 1999;14(7):1735-40.

28. Infertility: A comprehensive review. Hum Reprod 1998;13(4): 873-79.

29. Marshall LM, Spiegelman D, Goldman MB. A prospective study of reproductive factors and oral contraceptive use in relation to the risk of uterine leiomyomata. Fertil Steril 1998;70:432-39.

30. Seinera P, Farina C, Todros T. Laparoscopic myomectomy and subsequent pregnancy (results in 54 patients). Hum Reprod 2000;15:1993-96. 